\title{
The Need for Extracorporeal Membrane Oxygenation in Adults Undergoing Congenital Heart Surgery: Impact and Trends of Utilization
}

\author{
Salvatore Aiello, Rohit S. Loomba', Connor Kriz, Matthew Buelow', Saurabh Aggarwal' ${ }^{2}$, Rohit R. Arora \\ Division of Cardiology, Chicago Medical School, North Chicago, IL, 'Division of Cardiology, Children's Hospital of Wisconsin/Medical College of Wisconsin, \\ Milwaukee, WI, '2Division of Cardiology, Creighton University School of Medicine, Omaha, NE, USA
}

\section{Abstract}

Introduction: Adults with congenital heart disease (ACHD) represent a population with unique health-care needs. Many patients require cardiac surgery, with some requiring postoperative extracorporeal membrane oxygenation (ECMO). This study aimed to identify the risk factors for the need of postoperative ECMO and characterize the impact of ECMO on admission characteristics. Methods: Data from the 2005-2012 iterations of the Nationwide Inpatient Sample were used. ACHD admissions over 18 years with a documented cardiac surgery were included. Univariate analysis was conducted to compare the characteristics between those requiring ECMO and those who did not. Regression analysis was done to identify the independent risk factors associated with ECMO and to determine the impact of ECMO on length, cost, and mortality of the admission. Results: A total of 186,829 admissions were included. Of these, 446 (0.2\%) admissions required ECMO. Those with acute kidney injury, double-outlet right ventricle, or total anomalous pulmonary venous connection were more likely to require ECMO. ECMO was also significantly more utilized in patients undergoing septal defect repair, complete repair of tetralogy of Fallot, atrial switch, and heart transplant. The use of ECMO significantly increased length, cost, and mortality of stay. Overall mortality was $62.6 \%$ in the ECMO group. Conclusion: ECMO is only needed in a small proportion of postoperative ACHD patients. The use of ECMO significantly increases cost, length of stay and mortality in these patients. Improved identification of postoperative ACHD patients who are more likely to survive ECMO may facilitate improved survival and decreased resource utilization.

Keywords: Adult congenital heart disease, extracorporeal membrane oxygenation, mechanical support

\section{INTRODUCTION}

Patients with congenital heart disease (CHD) are living longer and increasingly surviving into adulthood. ${ }^{[1]}$ Currently, nearly $60 \%$ of all CHD patients are adults and recent measurements of the adult $\mathrm{CHD}$ (ACHD) patient population range from 1.47 to 3 per thousand.$^{[1-3]}$ The ACHD patient population is expected to continue growing until 2050.$^{[3]}$ As the ACHD patients age, treatments increasingly require emergency department evaluation, hospitalization, and elective or emergent surgery. ${ }^{[4]}$

ACHD patients who undergo surgery require a disproportionate allocation of resources. ${ }^{[5]}$ Increased resource utilization in the ACHD appears to coincide with an overall increased postoperative morbidity and mortality. ${ }^{[5,6]}$

While many ACHD patients' conditions require cardiac surgery, only a small percentage of these patients require

\begin{tabular}{|l|l|}
\hline \multicolumn{2}{|c|}{ Access this article online } \\
\hline Quick Response Code: & Website: \\
\hline & www.ijccm.org \\
\hline & \\
\hline
\end{tabular}

postoperative extracorporeal membrane oxygenation (ECMO) to support circulation while the heart recovers. ${ }^{[7]}$ There is a paucity of data regarding postoperative ACHD patients and the need for ECMO as well as ECMO survival..$^{[7,8]}$ There are also limited data regarding the risk factors associated with the need for ECMO and quantification of the impact ECMO has on admission length and cost.

We conducted an analysis of the Nationwide Inpatient Sample (NIS), a national administrative discharge database, with a primary aim of identifying risk factors associated with

Address for correspondence: Dr. Salvatore Aiello, Chicago Medical School, 3333 North Chicago, IL, USA. E-mail: salvatore.aiello@my.rfums.org

This is an open access article distributed under the terms of the Creative Commons Attribution-NonCommercial-ShareAlike 3.0 License, which allows others to remix, tweak, and build upon the work non-commercially, as long as the author is credited and the new creations are licensed under the identical terms.

For reprints contact: reprints@medknow.com

How to cite this article: Aiello S, Loomba RS, Kriz C, Buelow M, Aggarwal S, Arora RR. The need for extracorporeal membrane oxygenation in adults undergoing congenital heart surgery: Impact and trends of utilization. Indian J Crit Care Med 2017;21:547-51. 
ECMO utilization in ACHD patients undergoing cardiac surgery. Secondary aims included assessing trends in utilization of ECMO over an 8-year period and determine the impact of ECMO on admission characteristics.

\section{Methods}

\section{Patient identification}

A retrospective cohort study was conducted using the NIS. Data regarding hospital admissions were obtained from the 2005-2012 NIS iterations. Only admissions of patients over 18 years of age with CHD undergoing heart surgery were included. These were identified using ICD-9 diagnosis and procedure codes. Institutional review board approval was waived as the analyses utilized de-identified data from a national database.

CHD was identified using ICD-9 codes: double-outlet right ventricle using 745.11, atrioventricular septal defect using 745.60 , partial anomalous pulmonary venous connection using 747.42, total anomalous pulmonary venous connection using 747.41, transposition of the great arteries using 745.10 , congenitally corrected transposition using 745.12 , hypoplastic left heart syndrome using 746.7, atrial septal defect using 745.61, ventricular septal defect using 745.5, pulmonary atresia with ventricular septal defect using 746.01, tricuspid atresia using 746.1, Ebstein's anomaly using 746.2, truncus arteriosus using 745.0, and coronary artery anomaly using 746.85. Arrhythmias were identified using codes $427.0-427.42$ as well as $426.0-426.13$. It was not possible to distinguish between those with absence of a spleen or multiple spleens due to the ICD-9 coding strategy.

Cardiac surgery was identified using the following codes: codes 35.10 through 35.14 for valvuloplasty with no valve replacement, 35.20 through 35.28 for valvuloplasty with replacement, codes 35.50 through 35.73 for septal defect repair (including atrioventricular septal defect repair), 35.81 for complete repair of tetralogy of Fallot, 35.82 for pulmonary venous repair, 35.83 for complete repair of common arterial trunk, 35.84 for arterial switch operation for transposition, 35.91 for atrial switch operation for transposition, 35.92 for right ventricle to pulmonary artery conduit, 39.22 for Blalock-Tausig shunt, and 37.51 for heart transplant.

ECMO was identified using ICD-9 code 39.65.

\section{Data identification and collection}

Demographic information including gender and race were collected for each admission. Comorbid conditions and admission characteristics such as admission month, length of stay, and cost of stay were collected and assessed.

\section{Statistical analysis}

Continuous variables are reported using mean and standard deviation while categorical variables are reported using absolute frequency and percentages. Continuous variables were analyzed using a student $t$-test or Mann-Whitney U-test as appropriate with categorical variables being analyzed using Chi-square analysis. Baseline characteristics such as age, gender, race, and comorbid conditions were compared between those with and without ECMO.

Next, the proportion of admissions requiring ECMO, length of ECMO admissions, cost of ECMO admissions, and mortality of ECMO admissions was compared by the years from which data were included using an analysis of variance.

Logistic regression was then conducted to determine factors associated with need for ECMO with ECMO as the dependent variable. Next, a logistic regression was done to determine risk factors for inpatient mortality with inpatient mortality as the dependent variable and ECMO included as an independent variable. Linear regression analyses with length of stay and cost of admission as independent variables to determine the effect were then conducted to determine the impact of ECMO on these variables.

All statistical analyses were done using SPSS version 20.0 (IBM, Chicago, IL, USA).

\section{RESULTS}

Admission characteristics in those with and without extracorporeal membrane oxygenation (univariate analysis)

A total of 186,929 admissions with ACHD and a documented cardiac surgery were included in the analysis. Of these, $446(0.2 \%)$ admissions required ECMO. Those requiring ECMO were significantly more likely to be younger (56.1 years vs. 65.6 years, $P<0.001$ ) and of male gender (63.5\% vs. $58.3 \%, P=0.029)$. Racial differences were also present with those requiring ECMO more likely to be African American, Hispanic, or Asian/Pacific Islander $(P<0.01)$. Comorbidities more likely in those with ECMO included heart failure (odds ratio [OR] 2.4, 95\% confidence interval [CI] 2.0-2.9, $P<0.001$ ) and acute kidney injury (OR 8.9. 95\% CI 7.4-10.8, $P<0.001$ ) [Table 1].

Length of stay was significantly longer in those with ECMO (23.9 days vs. 11.5 days, $P<0.001)$ as was cost of the admission $(\$ 642,410$ vs. $\$ 174,424, P<0.001)$. Inpatient mortality was also greater in those with ECMO with a $62.6 \%$ mortality in those with ECMO compared to $4.5 \%$ in those without (OR 35.1, 95\% CI 28.9-42.6, P<0.001) [Table 1].

\section{Cardiac morphology and surgery in those with and without extracorporeal membrane oxygenation}

The following cardiac malformations were more likely in those with ECMO: double-outlet right ventricle (OR 26.3, 95\% CI 8.1-84.8, $P<0.001)$, total anomalous pulmonary venous connection (OR 20.9, 95\% CI 2.8-156.5, $P<0.001$ ), ventricular septal defect (OR 2.3, 95\% CI 1.1-5.7, $P=0.049$ ), pulmonary atresia (OR 9.7, 95\% CI 1.3-70.9, $P=0.006$ ), and congenitally corrected transposition (OR 7.9, 95\% CI 1.1-57.3, $P=0.015)$ [Table 1]. 


\begin{tabular}{|c|c|c|c|c|}
\hline & No ECMO $(n=186,483)$ & $\operatorname{ECMO}(n=446)$ & OR $(95 \% \mathrm{CI})$ & $P$ \\
\hline Age (years) & $65.5 \pm 14.3$ & $56.1 \pm 15.8$ & - & $<0.001$ \\
\hline Female & 77,717 (41.7) & $163(36.5)$ & $0.8(0.6-0.9)$ & 0.029 \\
\hline \multicolumn{5}{|l|}{ Race } \\
\hline Caucasian & $123,246(80.7)$ & $259(71.3)$ & - & $<0.001$ \\
\hline African American & $10,192(6.7)$ & $45(12.4)$ & & \\
\hline Hispanic & $9964(6.5)$ & $27(7.4)$ & & \\
\hline Asian or Pacific Islander & $3093(2.0)$ & $12(3.3)$ & & \\
\hline Native American & $971(0.6)$ & $* * *(* * *)$ & & \\
\hline Other & $5177(3.4)$ & $18(5.0)$ & & \\
\hline Heart failure & $63,897(34.2)$ & $249(55.8)$ & $2.4(2.0-2.9)$ & $<0.001$ \\
\hline Tachyarrhythmia & $80,584(43.2)$ & $141(31.6)$ & $0.6(0.4-0.7)$ & $<0.001$ \\
\hline Atrioventricular block & $5823(3.1)$ & $* * *(* * *)$ & $0.2(0.1-0.6)$ & 0.003 \\
\hline Acute kidney injury & $29,532(15.8)$ & $280(62.8)$ & $8.9(7.4-10.8)$ & $<0.001$ \\
\hline \multicolumn{5}{|l|}{ Cardiac lesion } \\
\hline Double outlet right ventricle & $48(0.1)$ & $* * *(* * *)$ & $26.3(8.1-84.8)$ & $<0.001$ \\
\hline Atrioventricular septal defect & $200(0.1)$ & 0 & - & 0.489 \\
\hline Partial anomalous pulmonary venous connection & $183(0.1)$ & $* * *(* * *)$ & $2.3(0.3-16.4)$ & 0.393 \\
\hline Total anomalous pulmonary venous connection & $20(0.1)$ & $* * *(* * *)$ & $20.9(2.8-156.5)$ & $<0.001$ \\
\hline Coronary artery anomaly & $431(0.2)$ & $* * *(* * *)$ & $2.9(0.9-9.1)$ & 0.053 \\
\hline Atrial septal defect & $16,634(8.9)$ & $30(6.7)$ & $0.7(0.5-1.1)$ & 0.105 \\
\hline Tetralogy of Fallot & $252(0.1)$ & $* * *(* * *)$ & $3.3(0.8-13.4)$ & 0.073 \\
\hline Ventricular septal defect & $890(0.5)$ & $* * *(* * *)$ & $2.3(1.1-5.7)$ & 0.049 \\
\hline Pulmonary atresia & $43(0.1)$ & $* * *(* * *)$ & $9.7(1.3-70.9)$ & 0.006 \\
\hline Tricuspid atresia & $51(0.1)$ & 0 & - & 0.727 \\
\hline Ebstein anomaly & $140(0.1)$ & $* * *(* * *)$ & $2.9(0.4-21.4)$ & 0.251 \\
\hline Hypoplastic left heart syndrome & $* * *(* * *)$ & 0 & - & 0.905 \\
\hline Transposition & $37(0.1)$ & 0 & - & 0.766 \\
\hline Congenitally corrected transposition & $53(0.1)$ & $* * *(* * *)$ & $7.9(1.1-57.3)$ & 0.015 \\
\hline Common arterial trunk & $32(0.1)$ & 0 & - & 0.782 \\
\hline Valvar stenosis or insufficiency & $167,463(89.8)$ & $398(89.2)$ & - & 0.841 \\
\hline \multicolumn{5}{|l|}{ Cardiac surgery } \\
\hline Valvuloplasty, no valve replacement & $34,615(18.5)$ & $85(19.1)$ & $1.0(0.8-1.3)$ & 0.782 \\
\hline Valvuloplasty with valve replacement & $139,088(74.5)$ & $269(60.3)$ & $0.5(0.4-0.6)$ & $<0.001$ \\
\hline Septal defect repair & $18,902(10.1)$ & $58(13.0)$ & $1.3(1.1-1.7)$ & 0.044 \\
\hline Tetralogy of Fallot repair & $66(0.1)$ & $* * *(* * *)$ & $12.7(3.1-52.1)$ & $<0.001$ \\
\hline Total anomalous pulmonary venous connection repair & $127(0.1)$ & $* * *(* * *)$ & $3.3(0.4-23.6)$ & 0.208 \\
\hline Right ventricle to pulmonary artery conduit & $151(0.1)$ & $* * *(* * *)$ & $2.7(0.3-10.8)$ & 0.289 \\
\hline Blalock-tausig shunt & $4619(2.5)$ & $* * *(* * *)$ & $0.2(0.1-0.8)$ & 0.014 \\
\hline Common arterial trunk repair & $* * *(* * *)$ & 0 & - & 0.945 \\
\hline Arterial switch & $13(0.1)$ & 0 & - & 0.860 \\
\hline Atrial switch & $106(0.1)$ & $* * *(* * *)$ & $7.9(1.9-32.2)$ & 0.001 \\
\hline Heart transplant & $2834(1.5)$ & $90(20.2)$ & $16.4(12.9-20.7)$ & $<0.001$ \\
\hline Length of hospital stay (days) & $11.5 \pm 6.2$ & $23.9 \pm 11.9$ & - & $<0.001$ \\
\hline Cost of hospitalization (US dollars) & 174,424 & 642,410 & - & $<0.001$ \\
\hline Inpatient mortality & $8459(4.5)$ & $279(62.6)$ & $35.1(28.9-42.6)$ & $<0.001$ \\
\hline
\end{tabular}

The following cardiac surgeries were more likely in those with ECMO: septal defect repair (OR 1.3, 95\% CI 1.1-1.7, $P=0.044$ ), complete repair of tetralogy of Fallot (OR 12.7, 95\% CI 3.1-52.1, $P<0.001$ ), atrial switch (OR 7.9, 95\% CI 1.9-32.2), and heart transplant (OR 16.4, 95\% CI 12.9-20.7) [Table 1].
Trends in extracorporeal membrane oxygenation utilization and burden over the study period

Overall, ECMO was utilized in $0.2 \%$ of included admissions. The percentage of admissions in which ECMO was utilized increased significantly across years $(0.1 \%$ in $2005-0.6 \%$ in 2012, $P<0.001)$. Cost of admissions with ECMO also 
increased significantly over the study period with mean total charges of \$470,491 in 2005 compared to \$804,046 in 2012 $(P=0.001)$. Inpatient mortality and length of stay did not significantly change over this time period [Table 2].

\section{Independent risk factors associated with extracorporeal membrane oxygenation (regression analysis)}

The following risk factors were associated with ECMO: acute kidney injury (OR 7.3, 95\% CI 5.7-9.3), double-outlet right ventricle (OR 8.0, 95\% CI 1.5-42.6), total anomalous pulmonary venous connection (OR 18.0, 95\%CI 1.6-203.1), septal defect repair (OR 3.9, 95\%CI 2.4-6.1), complete repair of tetralogy of Fallot (OR 19.0, 95\% CI 1.7-208.6), atrial switch (OR 14.2, 95\% CI 2.2-89.3), heart transplant (OR 10.5, 95\% CI 6.6-16.6), and later year in the study period (OR 4.6, 95\% CI 2.6-8.0) [Table 3].

\section{Effect of extracorporeal membrane oxygenation on} admission characteristics (regression analysis)

After multivariate analysis, the use of ECMO was associated with significant increases in the length, cost, and mortality of admissions. ECMO increased the length of admission by 3.2 days, increased the cost of admission by approximately $\$ 343,694$, and increased inpatient mortality approximately 24-fold [Table 4].

\section{Discussion}

Our analysis of the NIS database demonstrated that only $0.2 \%$ of ACHD patients undergoing cardiac surgery require postoperative ECMO. However, during the study period, we found there to be an overall increasing proportion of ACHD admissions requiring postoperative ECMO with the most recent year having a $0.6 \%$ frequency. Particular subsets of patients were more likely to require postoperative ECMO: those with acute kidney injury, double-outlet right ventricle, or total anomalous pulmonary venous connection. Those undergoing septal defect repair, complete repair of tetralogy of Fallot, atrial switch, and heart transplant were also more likely to require postoperative ECMO.

The frequency of postoperative ECMO in our study was lower than that found in a previous study by Acheampong et al. who reported that $1.1 \%$ of ACHD patients required postoperative ECMO. While the overall frequency in our study was $0.2 \%$, it should be noted that this percentage was $0.6 \%$ when only the final study year is considered. This suggests a trend toward the values documented by Acheampong et al. Nonetheless, both studies highlight the low frequency of postoperative ECMO in this population. ${ }^{[7]}$
Furthermore, our study revealed that the use of ECMO increased the cost of admission by approximately $\$ 343,694$. Increased cost of admission may be attributed to the need for specialized care as well as increased length of stay required for ACHD patients. ${ }^{[5]}$ Our study demonstrates that the use of ECMO in ACHD patients increased the length of stay by over 3 days. Previous data of the impact of postoperative ECMO in ACHD patients are not available for comparison.

Our study also demonstrated that ACHD patients requiring postoperative ECMO have significantly increased mortality. In our study, only $62.6 \%$ of those requiring ECMO experience inpatient mortality. This high rate of mortality may be due to the degree of illness and number of comorbidities present in those who are placed on ECMO. ECMO-related complications are also likely to contribute to this high rate of mortality. It remains unclear at this time what the particular mechanisms of mortality are in those with ACHD requiring postoperative ECMO. A better delineation of these factors will perhaps allow for better prediction of those who have an increased likelihood of surviving to discharge after postoperative ECMO. ${ }^{[8,9]}$

Our mortality data are similar to that of previous studies. Paden et al. reported a 60\% mortality rate in an analysis of Extracorporeal Life Support Organization data from ACHD patients requiring ECMO ${ }^{[10]}$ In another study examining ACHD patients receiving ECMO after cardiogenic shock, Schmidt et al. reported a mortality of $68 \%$, although it should be noted that this study did not exclusively include operative patients. ${ }^{[8]}$

The decision to utilize ECMO in ACHD patients is often complicated by their underlying cardiac anatomy and existing comorbidities. ${ }^{[4,9]}$ For patients undergoing elective surgery, it is important to counsel them regarding the possibility, albeit low, of postoperative ECMO. Clinicians should work with patients and their families to determine the patient's desires in regard to the use of ECMO if the need arises. These efforts could help optimize resource allocation and the overall emotional burden of such admissions. ${ }^{[11]}$

Clinicians must also use current data to help guide the decision to utilize ECMO. The SAVE score, a novel predictive mortality model, incorporates 13 pre-ECMO variables for patients requiring ECMO after cardiogenic shock. An internal validation of this scoring method resulted in greater hospital survival (67\% vs. $42 \%) .{ }^{[8,10]}$ Such

Table 2: Trends of extracorporeal membrane oxygenation admissions by year

\begin{tabular}{|c|c|c|c|c|c|c|c|c|c|}
\hline & 2005 & 2006 & 2007 & 2008 & 2009 & 2010 & 2011 & 2012 & $P$ \\
\hline Requiring ECMO & $19(0.1)$ & $19(0.1)$ & $13(0.1)$ & $44(0.2)$ & $66(0.2)$ & $68(0.3)$ & $83(0.3)$ & $134(0.6)$ & $<0.001$ \\
\hline ECMO mortality & $12(63.2)$ & $14(73.7)$ & $7(53.8)$ & $33(75.0)$ & $35(53.0)$ & $46(67.6)$ & $56(67.5)$ & $76(56.7)$ & 0.164 \\
\hline ECMO length of stay (days) & 33.1 & 11.1 & 24.1 & 20.2 & 27.2 & 19.1 & 21.2 & 28.0 & 0.113 \\
\hline ECMO cost of stay & 470,491 & 299,912 & 474,843 & 580,106 & 611,984 & 552,376 & 635,340 & 804,046 & 0.001 \\
\hline
\end{tabular}

ECMO: Extracorporeal membrane oxygenation 


\section{Table 3: Regression results identifying factors independently associated with increased need for extracorporeal membrane oxygenation}

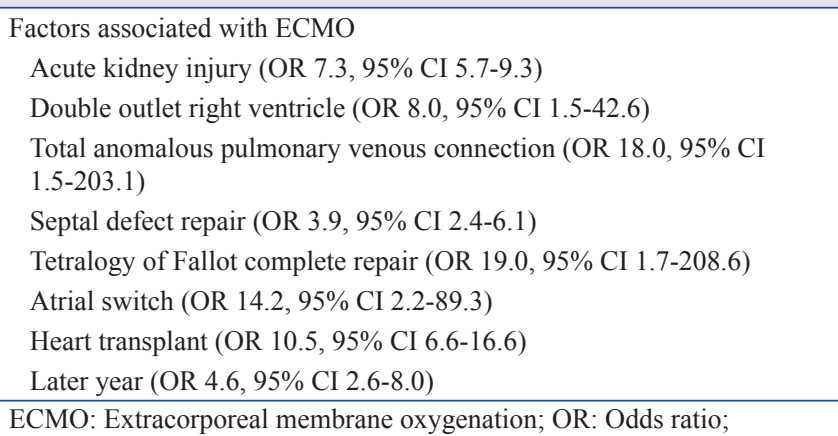
$\mathrm{CI}$ : Confidence interval

\section{Table 4: Regression results identifying impact of extracorporeal membrane oxygenation on admission characteristics}

\begin{tabular}{ll}
\hline Admission characteristic & Impact of ECMO \\
\hline Cost of admission & Increased by $\$ 343,694 ; P<0.001$ \\
Length of admission & Increased by 3.2 days; $P<0.001$ \\
Inpatient mortality & Increased (OR 23.7, $95 \%$ CI 18.5-30.4) \\
\hline
\end{tabular}

OR: Odds ratio; CI: Confidence interval

predictive models, specific to the ACHD population, may also be particularly helpful.

What must also be considered is the quality of life of those who survive to discharge. While patients may experience some decrease in physical and psychological aspects of their daily life, many of those who survive to discharge have been able to return to work or school and report good quality of life with the help of rehabilitation programs. ${ }^{[12-15]}$ It should be noted that this data does not come from the ACHD population.

A strength of this analysis is that it has a large number of patients as it utilizes data from a large national database. In addition, robust data regarding length and cost of admission are available from the NIS database. Despite the strengths of this analysis, there are also limitations. Several of the CHD ICD9 codes have not been validated. The coding for various congenital malformations of the heart may not accurate. It is also possible that the numbers in this study represent an underrepresentation although this should not impact the presence and degree of overall relation to the overall outcome of interest. In addition, this study is limited by data available in the database. Specifics regarding ECMO cannulation and duration are among data not available for inclusion in the analysis. Temporal associations cannot be made from these data and except for the CHD these are all associations.

Acknowledging the limitations, we feel that the findings of these analyses provide novel and valuable data regarding ECMO utilization and burden in adults with CHD during admissions for cardiac surgery.

\section{Conclusion}

Specific congenital heart malformations, cardiac surgeries, and comorbidities are associated with increased need for ECMO. Although needed in $<1 \%$ of ACHD admissions during which cardiac surgery is required, ECMO is associated with a significant increase in resource utilization and mortality.

\section{Financial support and sponsorship}

Nil.

\section{Conflicts of interest}

There are no conflicts of interest.

\section{RefEREnCES}

1. van der Bom T, Mulder BJ, Meijboom FJ, van Dijk AP, Pieper PG, Vliegen HW, et al. Contemporary survival of adults with congenital heart disease. Heart 2015;101:1989-95.

2. Marelli AJ, Ionescu-Ittu R, Mackie AS, Guo L, Dendukuri N, Kaouache $\mathrm{M}$, et al. Lifetime prevalence of congenital heart disease in the general population from 2000 to 2010. Circulation 2014;130:749-56.

3. Benziger CP, Stout K, Zaragoza-Macias E, Bertozzi-Villa A, Flaxman AD. Projected growth of the adult congenital heart disease population in the United States to 2050: An integrative systems modeling approach. Popul Health Metr 2015;13:29.

4. Agarwal S, Sud K, Menon V. Nationwide hospitalization trends in adult congenital heart disease across 2003-2012. J Am Heart Assoc 2016;5:43-54.

5. Bhatt $\mathrm{AB}$, Rajabali $\mathrm{A}, \mathrm{He} \mathrm{W}$, Benavidez OJ. High resource use among adult congenital heart surgery admissions in adult hospitals: Risk factors and association with death and comorbidities. Congenit Heart Dis 2015;10:13-20.

6. Rodriguez FH $3^{\text {rd }}$, Moodie DS, Parekh DR, Franklin WJ, Morales DL, Zafar F, et al. Outcomes of heart failure-related hospitalization in adults with congenital heart disease in the United States. Congenit Heart Dis 2013;8:513-9.

7. Acheampong B, Johnson JN, Stulak JM, Dearani JA, Kushwaha SS, Daly RC, et al. Postcardiotomy ECMO support after high-risk operations in adult congenital heart disease. Congenit Heart Dis 2016;11:751-5.

8. Schmidt M, Burrell A, Roberts L, Bailey M, Sheldrake J, Rycus PT, et al. Predicting survival after ECMO for refractory cardiogenic shock: The survival after veno-arterial-ECMO (SAVE)-score. Eur Heart J 2015;36:2246-56.

9. Uilkema RJ, Otterspoor LC. Extracorporeal membrane oxygenation in adult patients with congenital heart disease. Neth Heart J 2014;22:520-2.

10. Paden ML, Conrad SA, Rycus PT, Thiagarajan RR, ELSO Registry. Extracorporeal life support organization registry report 2012. ASAIO J 2013;59:202-10

11. Fernandes SM, Chamberlain LJ, Grady S Jr. Saynina O, Opotowsky AR, Sanders L, et al. Trends in utilization of specialty care centers in california for adults with congenital heart disease. Am J Cardiol 2015;115:1298-304

12. Anselmi A, Flécher E, Corbineau H, Langanay T, Le Bouquin V, Bedossa M, et al. Survival and quality of life after extracorporeal life support for refractory cardiac arrest: A case series. J Thorac Cardiovasc Surg 2015;150:947-54.

13. Chen KH, Tsai FC, Tsai CS, Yeh SL, Weng LC, Yeh LC, et al. Problems and health needs of adult extracorporeal membrane oxygenation patients following hospital discharge: A qualitative study. Heart Lung 2016;45:147-53

14. Mojoli F, Venti A, Pellegrini C, De Ferrari GM, Ferlini M, Zanierato M, et al. Hospital survival and long term quality of life after emergency institution of venoarterial ECMO for refractory circulatory collapse. Minerva Anestesiol 2013;79:1147-55.

15. Hsieh FT, Huang GS, Ko WJ, Lou MF. Health status and quality of life of survivors of extra corporeal membrane oxygenation: A cross-sectional study. J Adv Nurs 2016;72:1626-37. 